\title{
STRONG CONVERGENCE PROPERTIES OF SFT RINGS
}

\author{
John T. Condo \\ 748 Jamie Way \\ Atlanta, GA 30188 \\ Jim Coykendall \\ Department of Mathematics \\ North Dakota State University \\ Fargo, ND 58105-5075
}

\begin{abstract}
This paper investigates a strong convergence property of rings of formal power series where the coefficient ring has the strong finite type (SFT) property. In particular,

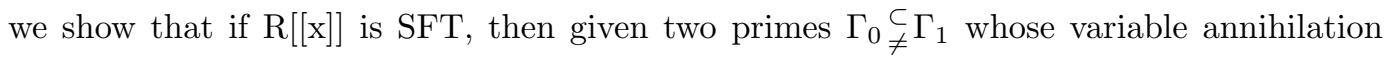
ideals agree, then $\mathrm{x} \in \Gamma_{1}$. We also show that this strong convergence property completely characterizes Noetherian rings.
\end{abstract}

\section{INTRODUCTION}

In this paper we assume that all rings are commutative with identity. In [1] Arnold defined the SFT property. We recall that an ideal $\mathrm{I} \subseteq \mathrm{R}$ is called an $S F T$ ideal if there is a finitely generated ideal $\mathrm{B} \subseteq \mathrm{I}$, and a fixed natural number $\mathrm{N}>0$ such that $\mathrm{a}^{\mathrm{N}} \in \mathrm{B}$ for every $\mathrm{a} \in \mathrm{I}$. We also say that $\mathrm{R}$ is an $S F T$ ring if every ideal $\mathrm{I} \subseteq \mathrm{R}$ (equivalently, every prime ideal) has the SFT property. Clearly every finitely generated ideal has the SFT property with $\mathrm{B}=\mathrm{I}$ and $\mathrm{N}=1$, so any Noetherian ring is trivially SFT. 
The SFT property is a property that was defined to highlight rings which have "bad power series dimension behavior"; that is, rings with finite Krull dimension such that the dimension of $\mathrm{R}[[\mathrm{x}]]$ is infinite. In [1] it was discovered that if $\mathrm{R}$ does not possess the SFT property, then $\operatorname{dim}(\mathrm{R}[[\mathrm{x}]])=\infty$, even if $\operatorname{dim}(\mathrm{R})=0$. Like coherence, the SFT property is an "almost Noetherian" property of a ring. It is of general interest to study such rings R, and in this paper we give an analytic connection between the ideal theory of $\mathrm{R}[\mathrm{x}]]$ and the convergence of formal power series in the x-adic topology.

In this paper, we investigate a Noetherian-like property which we call super-convergence in SFT power series rings $\mathrm{R}[[\mathrm{x}]]$. This property illustrates an analytic connection between the "formal" convergence of power series in the x-adic topology, and the ideal structure of $\mathrm{R}[[\mathrm{x}]]$. In particular, it is shown that if $\mathrm{R}[[\mathrm{x}]]$ is SFT, then given a Cauchy sequence of elements in a radical (e.g. prime) ideal $\mathrm{I} \subseteq \mathrm{R}[[\mathrm{x}]]$ (with respect to the metric induced by the x-adic topology) the limit of the sequence is also in I, or equivalently, infinite sums of elements of I are in I (provided that the sum converges x-adically). We shall see that this property implies that if $\mathrm{R}[[\mathrm{x}]]$ has the SFT property, then primes in a chain are almost distinguished by their image ideals in $\mathrm{R}$ under the variable annihilation map (in the sense that if $\Gamma_{0} \underset{\neq}{\subset} \Gamma_{1}$ are primes with equal image ideals, then $\mathrm{x} \in \Gamma_{1}$ ).

We also show that global super-convergence (super-convergence for every ideal of $\mathrm{R}[[\mathrm{x}]]$ ) gives a new characterization of Noetherian rings, and that radical super-convergence (superconvergence for each radical ideal) is a property possessed by every power series ring that is SFT.

We begin the investigation in the following section by deducing some of the consequences of the SFT property. 


\section{IMPLICATIONS OF THE SFT PROPERTY}

We begin by noting that an SFT ideal $\Gamma$ can be considered as an ordered triple $(\Gamma, \mathrm{B}, \mathrm{N})$ where $\Gamma$ is the SFT ideal, B is the finitely generated ideal contained in $\mathrm{I}$, and $\mathrm{N}$ is the natural number $\mathrm{N}>0$ such that $\mathrm{a}^{\mathrm{N}} \in \mathrm{B}$ for every $\mathrm{a} \in \Gamma$. In this paper we shall use this notation.

We note that the definition of SFT "almost" implies that $\mathrm{I}^{\mathrm{N}} \subseteq \mathrm{B}$. We shall presently make this more precise, and we shall enumerate some important cases where it is true that $\mathrm{I}^{\mathrm{N}} \subseteq \mathrm{B}$.

THEOREM 2.1 Let $\Gamma$ be an SFT ideal of a ring $\mathrm{R}$ with data $(\Gamma, \mathrm{B}, \mathrm{N})$. If $\left\{\alpha_{i}\right\}_{i=1}^{\mathrm{N}}$ denotes a collection of $\mathrm{N}$ elements of $\Gamma$, then

$$
\sum_{n_{1}+\ldots+n_{k}=\mathrm{N}} \frac{\mathrm{N} !}{n_{1} ! \ldots n_{k} !} \alpha_{1}^{n_{1}} \ldots \alpha_{k}^{n_{k}} \in \mathrm{B}
$$

Where $n_{j} \geq 1$ for every $j$. In particular, $\mathrm{N} ! \alpha_{1} \ldots \alpha_{\mathrm{N}} \in \mathrm{B}$.

Proof: For a fixed N, the above is true if $k=1$ by definition. We proceed by induction; fixing $\mathrm{N}$, we show an analogous formula for the case $k+1$ if $k \leq \mathrm{N}-1$.

We begin by replacing $\alpha_{k}$ in the above sum by $\alpha_{k}+\alpha_{k+1}$ which is also an element of Г. We obtain:

$$
\sum_{n_{1}+\ldots+n_{k}=\mathrm{N}} \frac{\mathrm{N} !}{n_{1} ! \ldots n_{k} !} \alpha_{1}^{n_{1}} \ldots \alpha_{k-1}^{n_{k-1}}\left(\alpha_{k}+\alpha_{k+1}\right)^{n_{k}} \in \mathrm{B}
$$

and so 


$$
\sum_{n_{1}+\ldots n_{k}=\mathrm{N}} \frac{\mathrm{N} !}{n_{1} ! \ldots n_{k} !} \alpha_{1}^{n_{1}} \ldots \alpha_{k-1}^{n_{k-1}}\left(\sum_{r=0}^{n_{k}} \frac{n_{k} !}{r !\left(n_{k}-r\right) !} \alpha_{k}^{r} \alpha_{k+1}^{n_{k}-r}\right) \in \mathrm{B} .
$$

Distributing the sum, we have

$$
\begin{aligned}
\sum_{n_{1}+\ldots+n_{k}=\mathrm{N}} & \frac{\mathrm{N} !}{n_{1} ! \ldots n_{k} !} \alpha_{1}^{n_{1}} \ldots \alpha_{k-1}^{n_{k-1}} \alpha_{k}^{n_{k}}+\sum_{n_{1}+\ldots n_{k}=\mathrm{N}} \frac{\mathrm{N} !}{n_{1} ! \ldots n_{k} !} \alpha_{1}^{n_{1}} \ldots \alpha_{k-1}^{n_{k-1}} \alpha_{k+1}^{n_{k}} \\
& +\sum_{n_{1}+\ldots+n_{k}=\mathrm{N}} \frac{\mathrm{N} !}{n_{1} ! \ldots n_{k} !} \alpha_{1}^{n_{1}} \ldots \alpha_{k-1}^{n_{k-1}}\left(\sum_{i+j=n_{k}} \frac{n_{k} !}{i ! j !} \alpha_{k}^{i} \alpha_{k+1}^{j}\right)
\end{aligned}
$$

where $i, j>0$.

By induction, the first two sums are in B, and the last one can be written in the form:

$$
\sum_{n_{1}+\ldots+n_{k-1}+i+j=\mathrm{N}} \frac{\mathrm{N} !}{n_{1} ! \ldots n_{k-1} ! i ! j !} \alpha_{1}^{n_{1}} \ldots \alpha_{k-1}^{n_{k-1}} \alpha_{k}^{i} \alpha_{k+1}^{j}
$$

which concludes the proof.

REMARK 2.2 The fact that $\mathrm{N} ! \alpha_{1} \ldots \alpha_{\mathrm{N}} \in \mathrm{B}$ can also be obtained as an application of Proposition 2, page 100, [3].

We shall now apply this theorem to see when the SFT data $(\Gamma, \mathrm{B}, \mathrm{N})$ satisfies the somewhat stronger condition $\Gamma^{\mathrm{N}} \subseteq \mathrm{B}$.

THEOREM 2.3 Let $\Gamma$ be an SFT ideal of R, a ring of characteristic zero, and let $\mathcal{P}$ be a prime minimal over $\Gamma$ such that $\mathcal{P} \cap \mathbf{Z}=(0)$. Then in the localization $\mathrm{R}_{\mathcal{P}}, \Gamma$ survives and $\left(\Gamma R_{\mathcal{P}}\right)^{\mathrm{N}} \subseteq \mathrm{BR}_{\mathcal{P}}$. In particular, if $\mathrm{R}$ contains the rational numbers, then all SFT ideals $\Gamma$ satify $\Gamma^{\mathrm{N}} \subseteq$ B. 
Proof It suffices to show the first statement, as the second remark follows easily. It is easy to see that in $\mathrm{R}_{\mathcal{P}}, \Gamma \mathrm{R}_{\mathcal{P}}$ is $\mathrm{SFT}$ with finitely generated ideal $\mathrm{BR}_{\mathcal{P}}$. Theorem 2.1 gives that for any collection $\left\{a_{1}, \ldots, a_{\mathrm{N}}\right\}$ of elements of $\Gamma \mathrm{R}_{\mathcal{P}}, \mathrm{N} ! a_{1} \ldots a_{\mathrm{N}} \in \mathrm{BR}_{\mathcal{P}}$. But since $\mathcal{P} \cap \mathbf{Z}=(0)$, this implies that $\mathrm{N}$ ! is a unit of $\mathrm{R}_{\mathcal{P}}$. This concludes the proof.

We remark that not every SFT ideal can be localized to an ideal that satisfies the above conditions. The next example illustrates this as well as giving a concrete example of a non-Noetherian, zero dimensional SFT ring.

EXAMPLE 2.4 Let $\mathrm{F}$ be a field of characteristic $p>0$. Consider the ring $\mathrm{R}=\mathrm{F}\left[x_{1}, x_{2}, \ldots\right] /\left(x_{1}^{p k}, x_{2}^{p k}, \ldots\right) . \mathrm{R}$ is a zero-dimensional, quasi-local (non-Noetherian) ring. $\mathrm{R}$ also has the SFT property. To see this note that for every $\mathrm{r}$ in the maximal ideal, $\mathrm{r}^{p k}=0$, by the binomial theorem. We also note that because of the fact that $\operatorname{char}(\mathrm{R})=p, p k$ ! times any collection of $p k$ elements of the maximal ideal is in $(0)$. We also note that it has been shown in $[1]$ that $\operatorname{dim}(\mathrm{R}[[\mathrm{t}]])=1$, and in $[4]$ it was shown that $\operatorname{dim}\left(\mathrm{R}\left[\left[\mathrm{t}_{1}, \ldots, \mathrm{t}_{n}\right]\right]\right)=\mathrm{n}$.

In contrast to the above example, we now consider an example from [6, Example 99], which is slightly in error, and we show how Theorem 2.1 helps us to avoid such errors.

EXAMPLE 2.5 Let $\mathrm{F}$ be a field of characteristic zero, and we consider the ring $\mathrm{R}=\mathrm{F}\left[x_{1}, x_{2}, \ldots\right] /\left(x_{1}^{N}, x_{2}^{N}, \ldots\right)$ where $N>1$. Let $\mathrm{y}_{i}$ be the image of $x_{i}$ in $\mathrm{R}$, and let $\mathrm{T}_{n}=\mathrm{R}\left[\left[t_{1}, \ldots, t_{n}\right]\right]$

As in Example 2.4, $\mathrm{R}$ is a zero-dimensional, quasi-local ring. However, for this example, $\operatorname{dim}\left(\mathrm{T}_{n}\right)=\infty$ for all $n \geq 1$. The key difference in this example is that $\mathrm{R}$ does not possess the SFT property, and to see this we shall apply Theorem 2.1.

It is easy to see that the maximal ideal of $\mathrm{R}$ is $\mathcal{M}=\left(\mathrm{y}_{1}, \mathrm{y}_{2}, \ldots\right)$, and that $\mathcal{M}$ is the radical 
of (0) (since every element of $\mathcal{M}$ is nilpotent). On the other hand, $\mathcal{M}^{k}$ is not the 0 -ideal for any $k>0$, as we can form arbitrarily long products of distinct $\mathrm{y}_{i}$ 's which are clearly nonzero. But by Theorem 2.3, since $\mathrm{R}$ contains the rational numbers, $\mathcal{M}^{k}$ must be (0) for some $k>0$, which is a contradiction. This is inconsistent with the statement in [6] that $\mathrm{T}_{1}$ is one dimensional.

\section{SUPER CONVERGENCE}

In this section, we investigate the strong convergence property in SFT power series rings. We begin with the following definition.

DEFINITION 3.1 We say that the ring $\mathrm{R}$ is $n$-stably $S F T$ if $\mathrm{R}\left[\left[x_{1}, \ldots, x_{n}\right]\right]$ is also SFT. Conventionally, 0-stably SFT means that R is SFT, and stably SFT means that R is n-stably SFT for all $\mathrm{n}$.

REMARK 3.2 We note here that Noetherian rings are stably SFT, since power series rings over Noetherian rings are Noetherian. Another class of stably SFT rings are the finite dimensional Prufer domains with the SFT property (in particular finite dimensional discrete valuation rings). This follows from [2] where it was shown that if $\mathrm{R}$ is an $\mathrm{m}$ dimensional SFT Prufer domain, then $\operatorname{dim}\left(\mathrm{R}\left[\left[x_{1}, \ldots, x_{\mathrm{n}}\right]\right]\right)=\mathrm{mn}+1$.

In this section we will be primarily concerned with rings that are n-stably SFT for some $\mathrm{n}>0$.

The algebraist's notion of formal convergence in $\mathrm{R}[[\mathrm{x}]]$ is justified by topologizing $\mathrm{R}[[\mathrm{x}]]$ $\mathrm{x}$-adically. In this sense, every Cauchy sequence in the dense subring $\mathrm{R}[\mathrm{x}]$ converges to a 
limit in $\mathrm{R}[[\mathrm{x}]]$. There are, however, some subtle points to be considered when this analytic notion is meshed with the ideal theory of $\mathrm{R}[[\mathrm{x}]]$.

DEFINITION 3.3 We say the ideal $\Gamma_{\neq}^{\subset} \mathrm{R}[[\mathrm{x}]]$ has the super-convergence property if given a countable collection of elements $\left\{\gamma_{i}\right\}$ of $\Gamma$ such that $\sum_{i=0}^{\infty} \gamma_{i} x^{i}$ converges in the x-adic topology, then the sum is in $\Gamma$.

EXAMPLE 3.4 Let $\mathrm{I}$ be a finitely generated ideal in $\mathrm{R}[[\mathrm{x}]]$, say $\mathrm{I}=\left(f_{1}, \ldots, f_{n}\right)$, and consider $\sum_{i=0}^{\infty} \gamma_{i} x^{i}$ where each $\gamma_{i} \in \mathrm{I}$.

We write $\gamma_{i}=\sum_{j=0}^{n} r_{i, j} f_{j}$, with $r_{i, j} \in \mathrm{R}[[\mathrm{x}]]$.

We note that

$$
\begin{gathered}
\sum_{i=0}^{\infty} \gamma_{i} x^{i}=\sum_{i=0}^{\infty} x^{i}\left(\sum_{j=1}^{n} r_{i, j} f_{j}\right) \\
=f_{1}\left(\sum_{i=0}^{\infty} r_{i, 1} x^{i}\right)+\ldots+f_{n}\left(\sum_{i=0}^{\infty} r_{i, n} x^{i}\right)
\end{gathered}
$$

which shows that the above sum is in I.

We note here that the above example shows that every ideal in a Noetherian ring has the super-convergence property. In contrast to the above situation, we shall look at another example.

EXAMPLE 3.5 Let $\mathrm{R}, \mathrm{T}_{n}$, and $\mathrm{y}_{i}$ be as in Example 2.5. Let $\mathcal{M}=\left(\mathrm{y}_{1}, \mathrm{y}_{2}, \ldots\right)$ and consider $\mathcal{M R}[[\mathrm{x}]] \subset \mathcal{M}[[\mathrm{x}]]$. The element $\sum_{i=0}^{\infty} \mathrm{y}_{i} x^{i}$ is an element of $\mathrm{R}[[\mathrm{x}]]$ such that for every $\mathrm{N}>0$, $\sum_{i=0}^{\mathrm{N}} \mathrm{y}_{i} x^{i} \in \mathcal{M R}[[\mathrm{x}]]$, but $\sum_{i=0}^{\infty} \mathrm{y}_{i} x^{i}$ is not even in the radical of $\mathcal{M} \mathrm{R}[[\mathrm{x}]]$. To see that this is true, note that if $\mathrm{f}(\mathrm{x})$ is in the radical of $\mathcal{M R}[[\mathrm{x}]]$, then for some positive integer $N$, 
$\mathrm{f}^{N} \in \mathcal{M R}[[\mathrm{x}]]$, that is

$$
(\mathrm{f}(\mathrm{x}))^{N}=\sum_{i=0}^{k} r_{i} m_{i}
$$

with $r_{i} \in \mathrm{R}[[\mathrm{x}]]$ and $m_{i} \in \mathcal{M}$. This implies that $\mathrm{f}(\mathrm{x})$ is nilpotent, but we know from [5] that $\sum_{i=0}^{\infty} \mathrm{y}_{i} x^{i}$ is not nilpotent, and this contradiction verifies our claim.

Here we give a theorem that shows that "global super-convergence", that is, superconvergence for every ideal in $\mathrm{R}[[\mathrm{x}]]$ is a characterization of Noetherian rings. We say that $\mathrm{R}[[\mathrm{x}]]$ is globally super-convergent if every ideal of $\mathrm{R}[[\mathrm{x}]]$ has the super-convergence property.

THEOREM 3.6 The following conditions are equivalent:

1. $\mathrm{R}[[\mathrm{x}]]$ is globally super-convergent.

2. For any finite set of indeterminates $\mathrm{R}\left[\left[\mathrm{x}_{1}, \ldots, \mathrm{x}_{n}\right]\right]$ is globally super-convergent.

3. $\mathrm{R}$ is Noetherian.

Proof $3 . \Longrightarrow 1$. is clear from Example 3.4. To see that $1 . \Longrightarrow 3$, we show the contrapositive.

Let $\mathrm{I}$ be a non-finitely generated ideal of $\mathrm{R}$ and let $\left\{a_{0}, a_{1}, \ldots\right\}$ be generators of $\mathrm{I}$ chosen so that $a_{k}$ is not in the ideal generated by $\left\{a_{0}, \ldots, a_{k-1}\right\}$. Consider the element $\sum_{i=0}^{\infty} a_{i} x^{i}$ of $R[[x]]$. Assume that this element is in $\operatorname{IR}[[x]]$. So we have 


$$
\sum_{i=0}^{\infty} a_{i} x^{i}=\sum_{i=0}^{k} a_{i} f_{i}(x)
$$

with $f_{i}(x) \in \mathrm{R}[[\mathrm{x}]]$. But by assumption, $a_{k+1}$ cannot be in the ideal generated by $\left\{a_{0}, \ldots, a_{k}\right\}$, therefore, we have our desired contradiction.

Clearly, $2 . \Longrightarrow 1$. and $1 . \Longrightarrow 2$. by induction since $\mathrm{R}$ is Noetherian implies that $\mathrm{R}\left[\left[\mathrm{x}_{1}, \ldots, \mathrm{x}_{n}\right]\right]$ is Noetherian. This concludes the proof.

In Example 3.5 we see that $\sum_{i=0}^{\infty} \mathrm{y}_{i} x^{i}$ converges in the x-adic topology, but the sum is in a much larger ideal than the one containing the term of the defining Cauchy sequence. We now introduce a theorem to illustrate the mechanics of this effect.

THEOREM 3.7 Let $\Gamma$ be a radical ideal in $\mathrm{R}[[\mathrm{x}]]$ that is SFT, then $\Gamma$ has the superconvergence property.

Proof We wish to show that an arbitrary infinite sum $\sum_{i=0}^{\infty} \gamma_{i} x^{i}$ with $\gamma_{i} \in \Gamma$ converges to a limit in $\Gamma$. Consider the ideal $\Gamma[[y]]_{\neq}^{\complement} \mathrm{R}[[\mathrm{x}, \mathrm{y}]]$. As $\Gamma$ has the SFT property, this means that $\Gamma[[\mathrm{y}]] \subseteq \sqrt{\Gamma \mathrm{R}[[\mathrm{x}, \mathrm{y}]]}$. Therefore the sum $\gamma=\sum_{i=0}^{\infty} \gamma_{i} \mathrm{y}^{i}$ is in the radical of the ideal generated by $\Gamma$ in $\mathrm{R}[[\mathrm{x}, \mathrm{y}]]$, and hence we can write

$$
\gamma^{N}=\gamma_{k_{1}} r_{1}(\mathrm{x}, \mathrm{y})+\ldots+\gamma_{k_{m}} r_{m}(\mathrm{x}, \mathrm{y})
$$

where $\gamma_{k_{i}} \in \Gamma$ and $r_{i}(\mathrm{x}, \mathrm{y}) \in \mathrm{R}[[\mathrm{x}, \mathrm{y}]]$.

We now merely notice that under the image of the map $\phi_{\mathrm{y}}: \mathrm{R}[[\mathrm{x}, \mathrm{y}]] \longrightarrow \mathrm{R}[[\mathrm{x}]]$ defined by $\phi_{\mathrm{y}} \mathrm{f}(\mathrm{x}, \mathrm{y})=\mathrm{f}(\mathrm{x}, \mathrm{x})$, we obtain: 


$$
\gamma^{N}=\gamma_{k_{1}} r_{1}(\mathrm{x}, \mathrm{x})+\ldots+\gamma_{k_{m}} r_{m}(\mathrm{x}, \mathrm{x})
$$

This shows that $\gamma$ is in the radical of $\Gamma$, and is therefore, by assumption, in $\Gamma$.

We say that $\mathrm{R}[[\mathrm{x}]]$ is radically super-convergent if each radical ideal of $\mathrm{R}[[\mathrm{x}]]$ has the super-convergence property (for example, $\mathrm{R}$ is 1 -stably SFT).

COROLLARY 3.8 Let R[[x]] be radically super-convergent, and let $\phi$ be the variable annihilation map given by $\phi(\mathrm{f}(\mathrm{x}))=\mathrm{f}(0)$. If $\Gamma_{0} \underset{\neq}{\subset} \Gamma_{1}$ are two primes of $\mathrm{R}[[\mathrm{x}]]$ such that $\phi\left(\Gamma_{0}\right)=\phi\left(\Gamma_{1}\right)$, then $\mathrm{x} \in \Gamma_{1}$.

Proof Assume that $\mathrm{x}$ is not in $\Gamma_{1}$ and let $\gamma$ be an element of $\Gamma_{1} \backslash \Gamma_{0}$. As the image ideals of $\Gamma_{0}$ and $\Gamma_{1}$ are identical we can find an element $\xi_{0} \in \Gamma_{0}$ such that $\phi\left(\xi_{0}\right)=\phi(\gamma)$, and so we have $\gamma=\xi_{0}+\mathrm{x} \gamma_{1}$. The fact that $\mathrm{x}$ is not in $\Gamma_{1}$ implies that $\gamma_{1} \in \Gamma_{1}$. We continue this process to obtain

$$
\gamma=\sum_{i=0}^{\infty} \xi_{i} \mathrm{x}^{i}
$$

But the fact that $\Gamma_{0}$ has the super-convergence property implies that $\gamma \in \Gamma_{0}$, which is a contradiction.

Corollary 3.8 shows that if R is 1-stably SFT, then primes in a chain are distinguished by their images in the coefficient ring up to inclusion of the variable $\mathrm{x}$ (which is a potentially valuable piece of information when considering the dimension question of $\mathrm{R}[[\mathrm{x}]])$. This is wildly untrue in the non-SFT case, however. In fact if $\mathcal{P}$ is a non-SFT prime ideal, then 
there is an infinite chain of primes of $\mathrm{R}[[\mathrm{x}]]$ that all have image ideal $\mathcal{P}[1]$.

COROLLARY 3.9 Let R[[x]] be radically super-convergent, and let $\left\{f_{1}, f_{2}, \ldots\right\}$ be a countable collection of elements of $\mathrm{R}[[\mathrm{x}]]$. Then $\sum_{i=0}^{\infty} f_{i} x^{i} \in \sqrt{\left\{f_{1}, f_{2}, \ldots\right\}}$.

We remark that in Corollary 3.9, the convergence is absolute in the sense that rearrangements do not matter for convergence in the radical. It is also useful to note that this theorem is also untrue in the non-SFT case. The remark following the previous Corollary 3.8 also applies here [1].

\section{SUMMARY OF IMPLICATIONS}

In this section, we tie together a few loose ends by summarizing our results, and highlighting some open questions.

THEOREM 4.1 For the following seven hypotheses, we have the implications $1 . \Longleftrightarrow 2$. and $3 . \Longrightarrow 4 . \Longrightarrow 5 . \Longrightarrow 6 . \Longrightarrow 7$. We also have $2 . \Longrightarrow 3$. if $\operatorname{dim}(\mathrm{R})<\infty$.

1. $\mathrm{R}$ is Noetherian

2. $\mathrm{R}\left[\left[x_{1}, \ldots, x_{n}\right]\right]$ is globally super-convergent for all $n$.

3. $\operatorname{dim}\left(\mathrm{R}\left[\left[x_{1}, \ldots, x_{n}\right]\right]\right)<\infty$ for all $n$.

4. $\mathrm{R}$ is stably SFT. 
5. $\mathrm{R}$ is $n$-stably SFT for some $n>0$.

6. $\mathrm{R}\left[\left[x_{1}, \ldots, x_{n}\right]\right]$ is radically super-convergent.

7. $\mathrm{R}$ is $(n-1)$-stably SFT.

Proof All of the above implications follow from the previous section with the exception of $6 . \Longrightarrow 7$. To prove this, we will use the abbreviation $\mathrm{S}=\mathrm{R}\left[\left[x_{1}, \ldots, x_{n-1}\right]\right]$ and assume that $\mathrm{S}$ is not SFT. From [1], we have the existence of a prime ideal $\mathcal{P} \subset \mathrm{S}$ such that $\mathcal{P}[[\mathrm{x}]]$ strictly contains the radical of $\mathcal{P S}[[\mathrm{x}]]$. In fact the proof of Theorem 1 of [1] shows that there is a prime ideal $\Gamma$ strictly between $\mathcal{P} \mathrm{S}[[\mathrm{x}]]$ and $\mathcal{P}[[\mathrm{x}]]$. It is easy to see, however, (c.f. Example 3.5) that any element of $\mathcal{P}[[\mathrm{x}]]$ can be written as an infinite sum of elements of $\mathcal{P} \mathrm{S}[[\mathrm{x}]]$. Therefore, $\Gamma$ is not closed under infinite converging sums, and hence, $\mathrm{S}[[\mathrm{x}]]$ is not radically super-convergent. This establishes the theorem.

At this time, it is not known whether any of the above implications are reversable. It would be interesting to see a proof or counterexample. Another open question is, "if R is finite dimensional and SFT, is $\mathrm{R}[[\mathrm{x}]]$ finite dimensional?" Analogously we might ask, "if $\mathrm{R}[[\mathrm{x}]]$ is finite dimensional, is $\mathrm{R}\left[\left[\mathrm{x}_{1}, \ldots, \mathrm{x}_{n}\right]\right]$ finite dimensional for all $n$ ?" The key to this may lie in the concept of SFT-stability, since it is a necessary condition. This leads to the question, "if R is SFT, then is R[[x]] SFT?" Although it is true that the SFT property descends (that is, $\mathrm{R}[[\mathrm{x}]]$ is SFT implies that $\mathrm{R}$ is SFT), we pessimistically note that the well-established bad behavior of $\mathrm{R}[[\mathrm{x}]]$ when $\mathrm{R}$ is non-Noetherian seems to lend credence to the existence of a counterexample, and we conjecture that the answer to the question of finite dimensionality and SFT-stability is "no". 
As a final concluding remark, we note that if $\mathrm{R}$ is not SFT, then we know by the work of Arnold [1], that $\mathrm{R}[[\mathrm{x}]]$ has an infinite tower of primes. To see that $\mathrm{R}[[\mathrm{x}]]$ is not SFT we can construct a sequence of elements $\left\{a_{0}, a_{1}, a_{2}, \ldots\right\}$ such that $a_{k}^{k}$ is not in the ideal generated by the $\left\{a_{0}, a_{1}, \ldots, a_{k-1}\right\}$ (this is actually the original idea behind the SFT concept [1]). In $\mathrm{R}[[\mathrm{x}]]$, consider the infinite tower of primes and choose the $i$ th element in the $i$ th prime, but not in the $(i-1)$ th prime. This gives a sequence of elements such that no power of the $i$ th element is in the ideal generated by the previous elements. This establishes the remark of the previous paragraph that the SFT property "descends."

\section{REFERENCES}

1. J. T. Arnold, Krull dimension in power series rings, Trans. Amer. Math. Soc. $\mathbf{1 7 7}(1973), 299-304$.

2. J. T. Arnold, Power series rings with finite Krull dimension, Indiana Univ. Math. J. 31(1982), 897-911.

3. N. Bourbaki, "Algebra I," Springer-Verlag, New York, 1989.

4 J. T. Condo, J. Coykendall, and D. E. Dobbs, Formal power series rings over zerodimensional SFT-rings, Comm. Algebra 24(1996), 2687-2698.

5. D. Fields, Zero divisors and nilpotent elements in power series rings, Proc. Amer. Math. Soc. $27(1969), 427-433$.

6. H. Hutchins, "Examples of Commutative Rings," Polygonal Publishing House, Passaic, 1981.

7. I. Kaplansky, "Commutative Rings," University of Chicago Press, Chicago, 1974. 